\title{
Monopole Evolution in the Early Universe
}

\author{
Michiyasu Nagasawa \\ Department of Information Science, Faculty of Science, \\ Kanagawa University, Kanagawa 259-1293, Japan \\ E-Mail: nagasawa@info.kanagawa-u.ac.jp
}

\begin{abstract}
The formation and evolution of monopoles produced at $\mathrm{TeV}$ energy scales are considered. They can contribute to the generation of baryon number and may solve the cosmological problem. The dynamics of electroweak defects is completely different from that of conventional grand unification scale defects so that their number density should not be described by the scaling model.
\end{abstract}

At the various kinds of phase transitions in the early universe, cosmological defects may have been produced and might explain some of the unresolved cosmological problems. In this contribution, we take particular interest in a certain type of complex defect, strings which have monopole and anti-monopole pairs at its ends (Kephart \& Vachaspati 1996).

The main motivation why the string-monopole defects are employed here is that they might explain the baryon asymmetry problem. Since the electroweak string based on the standard model has been proved to have too low production rate so that the standard electroweak string scenario cannot provide the observational amount of baryon number (Nagasawa \& Yokoyama 1996). Then the alternative scenario has been proposed in which the ordinary topological string produced at TeV scale (Brandenberger \& Davis 1994) or the sphaleron bound state on the string (Soni 1997; Aulakh, Nagasawa, \& Soni 1999) plays an important role. Moreover, the monopole pair connected by an unstable string might be promising.

In the model which we have employed, at the first phase transition monopoles are produced and then the second phase transition occurs and strings appear between monopoles and anti-monopoles. Subsequently at the final phase transition below the electroweak scale, strings become unstable and disappear. Using the appropriate parameter values, the monopole mass, $m_{M}$, can be evaluated as

$$
m_{M} \sim 2400 \mathrm{GeV},
$$

which is much smaller than the GUT energy scale.

In order to estimate the resulting baryon number generation efficiency in this model, the string number density must be deduced. It could be calculated using the monopole number density at the string appearance. The motion velocity of the monopole, $v$, obeys the equation as

$$
m_{M} \dot{v}=-\mathcal{N}_{m} T^{2} v,
$$

where $\mathcal{N}_{m}$ is the degree number of the particles which interact with the monopole. This equation can be solved under reasonable approximations and the resulting 


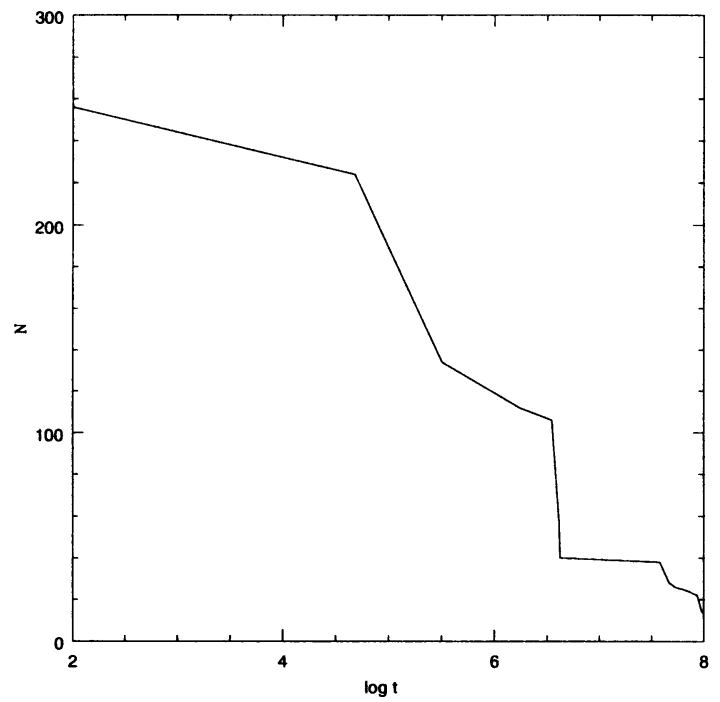

Figure 1. Time Evolution of Monopole Number

monopole number density at the string generation can be written as

$$
\frac{n_{M}}{s} \sim 10^{-16},
$$

where $s$ is the cosmological entropy density. Thus it may be difficult to produce sufficient baryon number by the dumbbell type complex defect. However, the fact that the monopole number density is very small means that the string production process would be approximately identical to the ordinary topological string case. Therefore the usual topological string loop baryogenesis scenario can be also applied to this model. Note that in contrast to the GUT monopole case $\left(m_{M} \sim 10^{16} \mathrm{GeV}\right)$, the cosmic energy density should not be dominated by monopoles in this model since the monopole mass is sufficiently light.

In the above, we have described a brief analytic check of the scenario. However, the basic feature of the defect interaction has been confirmed numerically by particle simulations. One example is shown in the Figure 1. Although this is only a preliminary calculation, further numerical simulations would be expected.

\section{References}

Kephart, T.W., \& Vachaspati, T. 1996, Phys.Lett.B388, 481

Nagasawa, M., \& Yokoyama, J. 1996, Phys.Rev.Lett77, 2166

Brandenberger, R.H., \& Davis, A.-C. 1994, Phys.Lett.B332, 305

Soni, V. 1997, Phys.Lett.B394, 275

Aulakh, C.S., Nagasawa, M., \& Soni, V. 1999, Phys.Lett.B471, 13 